DOI: https://doi.org/10.46630/phm.12.2020.16

Ivana M. Vilić ${ }^{1}$

Article de recherche

Université de Novi Sad

УДК 811.133.1'36:811.163.41'36

Faculté de philosophie

Reçu : le 18/2/2020

Département d'études romanes

\title{
ÉLÉMENTS EXTRALINGUISTIQUES DANS L'EXPRESSION DES RELATIONS SPATIALES EN FRANÇAIS ET EN SERBE
}

Les approches linguistiques cognitivistes soulignent le rôle des éléments extralinguistiques tels que la perception et l'organisation spatiale dans l'expression des relations spatiales. Dans cet article nous avons analysé l'impact de ces éléments en français et en serbe, notamment la taille de la cible et du site, la position des objets par rapport à la structure axiale et les propriétés dimensionnelles du site. Notre analyse basée sur la comparaison des moyens linguistiques dans les deux langues pour leur expression révèle beaucoup de ressemblances surtout en ce qui concerne la taille de la cible et du site et la localisation par rapport aux axes. Les deux langues divergent pourtant sur le point de dimensionnalité et de l'expression du mouvement dans l'espace.

Mots clés : relations spatiales, éléments extralinguistiques, préposition, cas, orientation dans l'espace, dimensionnalité, français, serbe

\section{Introduction}

Les éléments extralinguistiques représentent un facteur très important dans l'orientation dans l'espace et dans l'expression des relations spatiales. La localisation dans l'espace a intéressé surtout les linguistes d'orientation cognitiviste, vu que ce domaine comprend deux côtés - un côté linguistique, qui relève des moyens de son expression par la langue, mais aussi un côté lié à la perception visuelle et au système cognitif et conceptuel.

Le linguiste français Claude Vandeloise (1986) est l'un des plus connus qui ont introduit les concepts fonctionnels et les éléments extralinguistiques dans l'étude des prépositions spatiales, et qui a ouvert la voie à de nombreux travaux traitant les questions des relations spatiales en français (LAUR 1993 ; BORILLO 1998 ; AŠIĆ 2008, etc.). Dans la littérature serbe, les travaux de Milka Ivić (IVIĆ 1957 ; 1957-1958), qui a expliqué les traits pertinents des cas en serbe, surtout ceux dénotant les relations spatiales, représentent une

\footnotetext{
${ }^{1}$ ivavilic@ff.uns.ac.rs
} 
source inépuisable pour les recherches. Il faut mentionner aussi les travaux de Predrag Piper (PIPER 2001), qui offre une vision sémantique de l'expression des relations spatiales en serbe et dans d'autres langues slaves.

Le français et le serbe sont deux langues qui expriment les relations spatiales par des moyens linguistiques différents : le français utilise surtout les prépositions spatiales, alors que le serbe le fait par son système de cas, qu'ils soient sélectionnés ou non par des prépositions spatiales. La part des éléments extralinguistiques dans la localisation dans l'espace ne doit pas forcément être différente entre les deux langues, vu que ces éléments sont liés à la perception visuelle. C'est notre hypothèse de départ, que nous allons vérifier dans ce travail.

\section{La cible, le site et la préposition}

Dans l'expression linguistique de la localisation d'un objet dans l'espace, on remarque la présence de trois éléments nécessaires : l'objet à localiser, l'objet de référence, par rapport auquel la localisation se fait, et la préposition ${ }^{2}$. Dans la littérature française, les noms retenus pour désigner les deux objets sont la cible et le site (VANDELOISE 1986), alors que dans la littérature serbe on utilise les termes objekat lokalizacije et lokalizator (PIPER, ANTONIĆ et al. 2005). C. Vandeloise (1986), qui a été le premier à introduire les termes de cible et de site dans l'étude des relations spatiales en français, n'insiste pas explicitement sur la présence de préposition dans l'expression d'une relation spatiale, qui existe dans la définition serbe (PIPER, ANTONIĆ et al. 2005). La préposition, pourtant, est un élément qui exprime la relation entre la cible et le site de manière très claire, sans ambiguïtés ${ }^{3}$.

La définition des termes cible et site a pour origine les recherches dans la linguistique cognitive basées sur les notions gestaltistes de figure et de fond

\footnotetext{
${ }^{2}$ Dans la littérature linguistique française, les termes utilisés sont 'préposition' et 'locution prépositive' quand la préposition est composée de plusieurs mots. Dans notre travail, nous allons utiliser le terme de 'préposition', même pour les locutions prépositives.

${ }^{3}$ En parlant des prépositions spatiales en anglais, Landau \& Jackendoff (1993) constatent un phénomène linguistique intéressant : le nombre de prépositions spatiales en anglais est assez restreint par rapport au nombre de noms donnés aux objets. Dans la dénomination des objets, leurs caractéristiques géométriques précises sont prises en considération et on fait la différence entre un verre, une tasse, un bol, un bock, etc, ce qui n'est pas le cas dans la localisation dans l'espace et dans l'emploi des prépositions (en anglais). La même observation pourrait être faite pour le français et le serbe. Dans ces langues, les prépositions spatiales représentent des classes invariables de mots, dont le nombre n'est pas grand non plus, alors que, dans la dénomination des objets, on distingue les types d'objets par leurs caractéristiques physiques. L'explication de ce phénomène serait le fait que les prépositions spatiales expriment des relations fonctionnelles et que toute langue suit des règles spécifiques dans le domaine d'expression des relations spatiales.
} 
(TALMY 2000). C'est le principe de notre organisation cognitive qui est la base de l'explication de l'asymétrie existant dans l'expression de la relation entre un objet à situer (la cible) et une région dans laquelle cet objet est situé (le site). Cette région est le cadre qui contient et localise, alors que la cible représente le contenu, une entité matérielle qui occupe le volume de l'espace ${ }^{4}$ (TALMY $2000: 180$ ).

\section{La taille de la cible et du site}

Dans la perspective où les objets sont localisés par rapport à la région, les propriétés physiques des objets représentant le cadre et le contenu deviennent importantes. Le site, c'est-à-dire l'objet de référence, est en principe un objet plus grand, plus massif, stable et immobile, déjà connu des locuteurs, qui peut servir de point de repère. La cible, dont la position apporte une information nouvelle, est souvent un objet plus petit et mobile, qui, une fois entré dans la scène, devient plus saillant et perceptible.

Le principe de l'asymétrie des propriétés physiques de la cible et du site est présent en français (l'exemple 1) et en serbe (l'exemple 2) ${ }^{5}$ :

(1) La voiture est devant la maison.

* La maison est derrière la voiture.

(2) Automobil je ispred kuće.

*Kuća je iza automobila.

Ni le français ni le serbe n'admettent la possibilité de localiser un objet plus grand et stable (la maison) par rapport à un objet plus petit et mobile (la voiture). L'explication de l'asymétrie de la cible et du site se trouve probablement dans l'organisation de la cognition spatiale. La forme stable et connue du site comme objet de référence devrait faciliter la recherche de la cible et la navigation dans l'espace (LANDAU, JACKENDOFF 1993).

Il faut dire qu'il existe des prépositions en français et en serbe qui permettent l'inversion de la cible et du site, comme par exemple au-dessus del au-dessous de en français, iznad/ispod en serbe (exemples 3-4a) :

(3) Le projecteur est au-dessus de la table.

(3a) Projektor je iznad stola.

(4) La table est au-dessous du projecteur.

${ }^{4}$ Dans la définition de la cible et du site présentée par Talmy (2000), la notion de limite de l'objet localisé est très importante. C'est la limite de l'objet qui permet la distinction de la figure par rapport au fond.

${ }^{5}$ Le principe de l'asymétrie entre la cible et le site est marqué dans beaucoup de langues. Talmy (2000), qui a été le premier linguiste cognitiviste à approcher les études des relations spatiales aux études de la psychologie gestaltiste et de la perception visuelle, l'étudie en anglais. 
(4a) Sto je ispod projektora.

C. Vandeloise cite aussi les prépositions françaises avant/après qui admettent l'inversion de la cible et du site (VANDELOISE 1986). Nous reprenons ses exemples (5 et 6):

(5) Le banc est avant l'église.

(6) L'église est après le banc. (VANDELOISE 1986 : 36-37)

La traduction de ces exemples en serbe révèle le parallélisme entre les deux langues dans le cas des prépositions avant/après, en serbe pre/posle, par rapport à l'asymétrie de la cible et du site (exemples 5a et 6a):

(5a) Klupa je pre crkve.

(6a) Crkva je posle klupe.

La possibilité d'inversion de la cible et du site dans le cas des prépositions avant/après-pre/posle, ainsi que des prépositions au-dessus de/au-dessous deiznad/ispod s'explique par la relation entre la cible et le site. La taille de la cible et du site ne joue pas de rôle dans les exemples (3-6a) parce que les prépositions citées expriment une relation par rapport aux autres éléments présents dans cette relation, c'est-à-dire le mouvement et la position de la cible et du site sur la route du locuteur pour les prépositions avant/aprèspre/posle (exemples 5-6a), et l'orientation par rapport à l'axe vertical dans le cas des prépositions au-dessus de/au-dessous de-iznad/ispod (exemples 3-4a) (VANDELOISE 1986 : 36-37).

Le fait que les deux langues respectent les mêmes principes dans la localisation de deux objets par rapport à leur taille et qu'elles marquent précisément tous les éléments importants dans cette relation montre que la langue ne localise pas les objets absolument, mais le fait en respectant l'interrelation qui existe entre ces objets (LANDAU \& JACKENDOFF 1993 : 235).

\section{La structure axiale}

Un autre élément extralinguistique dans la localisation spatiale est la structure axiale. Les objets en tant que tels possèdent une orientation intrinsèque et on peut désigner le fond ou le dessus, le devant ou le derrière d'un objet. Cette orientation intrinsèque des objets se fait par rapport aux axes. Landau \& Jackendoff (1993) notent l'importance de plusieurs types d'axes : l'axe principal de l'objet - dans le cas du corps humain, c'est l'axe vertical, les axes d'orientation - par rapport au corps humain ce sont les axes horizontal et frontal, ainsi que les axes directionnels qui marquent les deux bouts de chaque axe.

Les deux langues, le français et le serbe, expriment assez précisément 
les relations par rapport à la structure axiale. En français, les prépositions qui expriment les relations par rapport aux directions précises - verticale, latérale et frontale, comme par exemple, au-dessus de-iznad, au-dessous deispod, à gauche de-levo od, à droite de-desno od, devant-ispred, derrière$i z a$, se distinguent sémantiquement et syntaxiquement des prépositions qui comprennent d'autres facteurs fonctionnels, comme par exemple, la position du locuteur par rapport à la cible et au site (avant-pre, après-posle), ou les relations porteur/porté (sur-na) ou contenant/contenu (dans-u). C. Vandeloise (1986 : 41-42) appelle le premier type les prépositions directionnelles et le deuxième type les prépositions fonctionnelles, soulignant les différences syntaxiques entre les deux types : dans le cas des prépositions directionnelles, le site est souvent introduit par la préposition de (La lampe est au-dessus de la table-Lampa je iznad stola).

En serbe, les relations liées à la structure axiale sont exprimées surtout par le cas génitif. C'est le cas par excellence pour désigner l'orientation par rapport à l'axe vertical-iznad $+N_{\text {Gén }} /$ ispod $+N_{\text {Gén }}$-au-dessus de / au-dessous de (Nosi suknju iznad kolena-Elle porte une jupe au-dessus du genou / Napisao je ime ispod crteža-Il a écrit son nom au-dessous du dessin), l'axe horizontal - desno od $+N_{\text {Gén }}$ /levo od $+N_{\text {Gén }}$ / pored $+N_{\text {Gén }}$-à droite de / à gauche de / à côté de (Kuhinja je desno od / levo od / pored dnevne sobe-La cuisine est à droite / à gauche / à côté du salon), ainsi que l'axe frontal - ispred $+N_{\text {Gén }}$ / iza $+N_{\text {Gén }}$-devant / derrière (On je ispred / iza vas-Il est devant / derrière vous). Le génitif dénotant l'orientation par rapport aux directions axiales en serbe s'oppose à l'instrumental qui, lui aussi, se combine avec les prépositions indiquant l'orientation par rapport aux axes : nad $+N_{\text {Instr }} /$ pod $+N_{\text {Instr }} /$ pred $+N_{\text {Instr }} / z a+N_{\text {Instr }}$-au-dessus de / sous / devant / derrière (Oblaci su nad gradom-Il y a des nuages au-dessus de la ville / Nosi novine pod miškom-Il porte un journal sous le bras / Stoji pred komisijom-Il est debout devant le jury / Za kućom je bašta-Il y a un jardin derrière la maison). La linguiste serbe M. Ivić explique la différence entre le génitif et l'instrumental en spécifiant que le génitif marque la position d'un objet par rapport aux points de limites, alors que l'instrumental précédé de préposition indique la relation d'orientation de la cible par rapport au site ${ }^{6}$ (IVIĆ 1957-58 : 151). La différence entre le génitif

${ }^{6}$ L'instrumental dénotant le lieu s'oppose aussi au locatif en serbe. Le locatif précise l'endroit de l'action verbale, comme dans l'exemple de M. Ivić Stoji na klupi-Il est debout sur le banc, alors que le site exprimé par l'instrumental dans Stoji nad klupom-Il est penché au-dessus du banc indique l'orientation par rapport à laquelle on situe l'action verbale (IVIĆ 1957 : 151). En parlant de l'opposition l'instrumental/le locatif dans les exemples cités ci-dessus, il faut souligner le rôle des prépositions : le locatif précédé de préposition na-sur exprime aussi un rapport plutôt fonctionnel de porteur/porté et la présence de contact entre la cible et le site, alors que dans le cas de préposition nad-au-dessus de devant le nom à l'instrumental (nad klupomau-dessus $d u$ banc) la notion de contact est absente. 
et l'instrumental en serbe est illustrée par les exemples (7-9) :

(7) Počešati se ispod kolena.

(Se gratter au-dessous du genou.)

(8) Počešati se pod kolenom.

(Se gratter sous le genou.)

(9) Cinq degrés au-dessous de zéro. (*sous zéro)

Pet stepeni ispod nule. (*pod nulom)

(10) « Vingt mille lieues sous les mers » (*au-dessous des mers)

„Dvadeset hiljada milja pod morem” (*ispod mora)

Dans l'exemple (7) le site (koleno-le genou) est exprimé par le génitif parce que le point représentant la cible, l'endroit où se gratter, est localisé par rapport à l'axe vertical sur lequel se trouve aussi le point représentant le site, le genou, et que les deux points sont conçus comme deux points de limites. La même relation est présente dans l'exemple (9), ce qui est exprimé par le génitif en serbe et la préposition française au-dessous de (ispod nuleau-dessous de zéro). Les exemples (8 et 10) illustrent toujours l'orientation par rapport à l'axe vertical, mais sans conceptualisation de la cible et du site comme des points de limites. Le site (koleno-le genou, l'exemple (8), morela mer l'exemple (10)) représente le lieu d'orientation par rapport auquel on situe la cible ou l'action verbale, ce qui est exprimé par l'instrumental en serbe et la préposition sous en français.

Les axes vertical, horizontal et frontal qui indiquent l'orientation intrinsèque d'un objet peuvent être considérés comme directionnels, c'està-dire comme possédant des limites de chaque côté. Les bouts de chaque axe marquent ainsi les bouts de l'objet même et permettent de distinguer le dessus et le fond ou le devant et le derrière d'un objet. Ce phénomène, qui est important pour la représentation mentale de la forme des objets, est aussi marqué linguistiquement. Les deux langues, le français et le serbe, disposent d'un nombre considérable de moyens linguistiques pour exprimer les relations liées aux limites des axes : au fond de-u dnu $+N_{\text {Gén' }}$, au bout de-nakraj $+N_{G e ́ n}$ $u k r a j+N_{G e ́ n}$, mais les prépositions par excellence pour marquer les points de limites de chaque côté de l'axe sont les prépositions de, depuis / à, jusque$o d, i z$ / do qui sont en serbe suivies par le nom au génitif, ${ }^{7}$ comme dans les exemples (11-12) :

(11) La plage s'étire depuis le village jusqu'aux rochers. Plaža se prostire od sela do stena.

(12) Être mouillé de la tête aux pieds. Biti mokar od glave do pete.

\footnotetext{
${ }^{7}$ Les prépositions de, depuis / à, jusque-od / do sont passées dans le domaine de l'expression temporelle où elles marquent aussi des périodes de temps bornées (cf. AŠIĆ : 2008).
} 
Dans l'exemple (11) les deux bouts sur l'axe horizontal sont marqués par les prépositions depuis (le village) - jusqu'aux (rochers) en français, et par les prépositions od (sela) - do (stena) en serbe. Pratiquement, les mêmes prépositions sont utilisées pour désigner les deux bouts sur l'axe vertical (exemple 12) : de (la tête) aux (pieds) en français, od (glave) do (pete) en serbe. Dans ce cas-là, une fois encore, c'est le génitif en serbe qui est utilisé, vu que les deux bouts représentent les points de limites.

\section{Les propriétés dimensionnelles du site}

La dimensionnalité en tant que propriété géométrique de l'espace a souvent été prise comme élément essentiel dans l'analyse des prépositions spatiales en français. G. Gougenheim (1949) explique les caractéristiques dimensionnelles des prépositions dans-u et sur-na, deux prépositions françaises par excellence dans l'expression des propriétés dimensionnelles du site : «Dans est la préposition de l'espace à trois dimensions, appréhendé non comme une surface, mais avec tout ce qui le remplit. Sur est la préposition de l'espace à deux dimensions, vu dans sa nudité, et non pas seulement comme une surface horizontale ; c'est la préposition de l'adhérence. » (GOUGENHEIM 1949 : 52). Suivant la ligne cognitiviste, C. Vandeloise (1986) critique une telle approche et insiste sur les concepts fonctionnels de porteur/porté pour la préposition sur-na et de contenant/contenu pour la préposition dans- $u$, acceptant néanmoins le fait que la dimensionnalité n'est souvent «qu'une conséquence superficielle » (VANDELOISE $1986: 15$ ).

Dans son article consacré aux aspects dimensionnels des prépositions françaises sur-na et dans-u, G. Gougenheim cite les facteurs extralinguistiques qui sont présents dans le choix de préposition sur-na ou dans-u en français. C'est surtout l'idée de rapport d'intériorité qui détermine le choix de la préposition dans-u -dans la commode-u komodi ou la position extérieure de superposition dans le cas de sur-na - sur la commode-na komodi. L'intériorité peut être conçue de manière plus abstraite, comme une sorte de cavité qui ne doit pas être fermée tout à fait - dans la poche-u džepu, dans les bras-u naručju. La notion de délimitation de l'espace contribue aussi au choix de la préposition dans-u-dans le jardin-u bašti, même quand la limite est vague ou qu'elle n'existe pas réellement - dans les eaux-u vodi, dans la terre-u zemlji, dans le miroir-u ogledalu (Gougenheim 1949).

Dans la littérature serbe, la dimensionnalité n'apparaît pas comme élément essentiel dans l'analyse des relations spatiales. Elle est pourtant présente dans l'analyse du cas locatif qui est précédé surtout de prépositions $u$-dans et na-sur. Ces deux prépositions sont définies comme marquant un 
rapport d'intériorité - u-dans et de la surface - na-sur (IVIĆ 1957 ; PIPER, ANTONIĆ et al. 2005). M. Ivić ajoute que les deux autres prépositions qui peuvent précéder un nom au locatif, les prépositions $o$ et $p o$ définissent elles aussi une idée spécifique de surface : la préposition po exprime que c'est la partie superficielle de la région qui est prise en considération, alors que la préposition $o$ dénote la forme ronde de la surface en question ${ }^{8}$ (IVIĆ 1957 : 152).

Le fait que les deux langues, le français et le serbe, utilisent pratiquement les mêmes prépositions pour exprimer les propriétés dimensionnelles du site, les prépositions sur en français, $n a(o, p o)$ en serbe pour marquer le caractère bidimensionnel du site et l'idée de surface ou dans, c'est-à-dire $u$ pour désigner l'espace tridimensionnel conçu comme intériorité ou contenant, ne devrait pas étonner. Du point de vue cognitiviste, le site ou l'objet de référence dans lequel la cible est située représente une région qui a un rôle très important dans l'organisation mentale de l'espace et dans l'orientation dans l'espace. Landau \& Jackendoff (1993), en se basant sur l'analyse des prépositions spatiales en anglais, émettent l'hypothèse, qui aurait une extension universelle, que la langue trie les éléments pertinents pour l'orientation dans l'espace. Les catégories de surface ou de volume seraient conservées en anglais comme dans beaucoup de langues et, comme on peut constater, en français et en serbe aussi ${ }^{9}$.

Le français possède encore une préposition dont la signification est liée au trait de dimensionnalité. Il s'agit de la préposition à qui dénote l'idée que le site est conçu de manière ponctuelle, c'est-à-dire que les propriétés

\footnotetext{
${ }^{8}$ Définissant les prépositions serbes $p o, n a, u$ T. Ašić relie la préposition $p o$ à la cible conçue comme " une substance continue ou une multitude d'objets », alors que la préposition $n a$ est réservée au cas où la cible est « un objet individuel ou plusieurs objets qui ont préservé leur individualité »(AŠIĆ 2008 : 183). Dans ses exemples que nous reprenons, la préposition serbe po est souvent traduite par la préposition sur-na, ce qui affirme qu'elle exprime la notion de surface : Puno časopisa je rasuto po stolu-Beaucoup de magazines sont éparpillés sur la table; Abi ima divne pegice po licu-Abi a de jolies tâches de rousseur sur le visage / Jedna velika knjiga je ležala na stolu-Un grand livre était posé sur la table; Tri knjige su ležale na stolu-Trois livres étaient posés sur la table (AŠIĆ 2008 : 184, 186). Nous nous arrêtons ici sur l'emploi statique de la préposition serbe po, vu que cette préposition est souvent utilisée avec les verbes dynamiques.

${ }^{9}$ Même si les deux langues utilisent pratiquement les mêmes prépositions pour exprimer les caractéristiques dimensionnelles des sites, la conceptualisation de l'espace comme bi- ou tridimensionnel en français et en serbe diffère souvent. L'idée de limite qui est présente dans la conceptualisation de l'espace tridimensionnel en français ne correspond pas toujours au cas corrélatifs en serbe. C'est ainsi qu'en français on dirait dans une île, dans une propriété, dans le ciel, alors qu'en serbe, dans ces cas-là, on utiliserait la préposition sur - na ostrvu, na imanju, na nebu. Il faut dire aussi que pour des syntagmes avec la préposition dans (dans la rue, dans l'herbe), le serbe peut utiliser les deux prépositions na-sur ou u-dans suivant le contexte : stanovati u ulici-habiter dans une rue, igrati se na ulici-jouer dans la rue; sedeti na travi-être assis dans l'herbe, izgubiti nešto u travi-perdre quelque chose dans l'herbe.
} 
dimensionnelles du site sont rendues à zéro. Même si le français et le serbe montrent des ressemblances sur beaucoup d'éléments extralinguistiques traités, la conceptualisation ponctuelle à laquelle renvoie la préposition à représente le point où les deux langues divergent considérablement.

En analysant les usages de la préposition à en français, C. Vandeloise (1988) relève ses caractéristiques dans l'expression de la localisation dans l'espace. Si les prépositions dans- $u$ et sur-na expriment les caractéristiques configurationnelles du site, « la préposition à, parce qu'elle a pour fonction essentielle la localisation de la cible par le site, maximalise l'asymétrie entre ces deux notions. » (VANDELOISE 1988 : 123) D'après cette constatation de C. Vandeloise, la préposition à aurait un rôle dans la spécification de la position du site et pour cette raison son usage comprend plusieurs contraintes. La préposition à est utilisée dans les cas où la position du site est différente de celle du locuteur et où le site est éloigné du lieu d'énonciation, ainsi que dans les cas où le site est hors du champ visuel du locuteur. Le site peut référer à la position du locuteur seulement dans les phrases à la première personne du singulier. Derrière ces contraintes, il y a l'idée de recherche qui est présente, et la préposition à en français dénote le chemin de recherche de la cible (VANDELOISE 1988 : 131-132). L'usage de la préposition à en français est illustré par les exemples (13-14) :

(13) Le footballeur est au terrain de football.

(13a) Fudbaler je na fudbalskom terenu.

(14) Le footballeur est sur le terrain de football.

(14a) Fudbaler je na fudbalskom terenu. (VANDELOISE $1988: 131$ )

L'exemple (13) pourrait être prononcé par un locuteur qui se trouve loin du terrain de football et pour lequel le site n'est pas présent dans son champ visuel. Dans le cas où le site (le terrain de football) est dans le champ visuel du locuteur, l'exemple (14) devient possible. La traduction de ces deux exemples (13a et 14a) montre qu'en serbe cette différence n'est pas marquée formellement.

D'un autre côté, les prépositions serbes na-sur et $u$-dans, qui sont elles aussi liées à la dimensionnalité, ont cette spécificité d'apparaître non seulement avec le locatif, mais aussi avec l'accusatif qui exprime un rapport différent par rapport au locatif. L'accusatif dans l'expression des relations spatiales en serbe dénote terminus ad quem de l'action verbale, alors que le locatif exprime le lieu de l'action dans sa totalité (IVIĆ $1957: 151)^{10}$. Les prépositions na-sur et $u$-dans marquent donc le point où l'action se termine (avec l'accusatif) ou

\footnotetext{
${ }^{10}$ L'opposition l'accusatif/le locatif dans le sens spatial en serbe est souvent expliquée par le trait de dynamisme : l'accusatif exprimerait le trait dynamique et le locatif le trait statique. Cette explication est erronée, souligne M. Ivić, en citant des exemples comme "jure se po bašti"-ils se coursent dans le jardin ou "trkaju se po poljani"-ils font la course dans le pré (IVIĆ 1957 $: 151)$.
} 
bien le lieu où l'action est située (avec le locatif), indiquant dans tous les deux cas les propriétés dimensionnelles du site (la surface ou le volume).

Un autre plan où les deux langues divergent serait donc lié à la notion de mouvement. La préposition $\grave{a}$ en français, avec sa conceptualisation ponctuelle, aide à définir le chemin de recherche de la cible (VANDELOISE 1988), alors que l'opposition entre l'accusatif et le locatif en serbe exprime aussi d'une manière le chemin parcouru vers le site, vu que ces deux cas désignent le lieu de l'action entière (le locatif) ou le lieu du moment final de l'action (l'accusatif) (IVIĆ 1957). Si l'accusatif marque le point final de l'action, ce point doit être atteint, ce qui introduit la notion de mouvement dans l'analyse des relations spatiales.

\section{L'aspect dynamique de localisation dans l'espace}

$\mathrm{Vu}$ que l'analyse présentée ci-dessus a été surtout orientée vers les relations statiques, la question qui peut être posée est celle de l'impact du mouvement et de son expression en français et en serbe. Sans entrer dans une analyse plus détaillée qui demanderait plus d'espace, nous pouvons seulement constater que, sur le plan formel, le français et le serbe diffèrent du point de vue de l'expression du mouvement dans l'espace.

Dans son article consacré à la relation entre le verbe et la préposition dans l'expression du déplacement en français, D. Laur (1993) constate qu'il existe une interrelation entre les verbes de déplacement et les prépositions. La combinatoire des traits sémantiques des deux classes obéit à des règles précises, mais c'est surtout le verbe qui exprime la signification de déplacement ou de changement de lieu, alors que la préposition qui suit le verbe détermine plutôt la localisation interne ou externe de la cible (on peut dire entrer dans ou sortir de, mais non *entrer contre ou *sortir autour) (LAUR 1993 : 65). En serbe, par contre, l'accusatif et le locatif marquent l'opposition entre l'aboutissement du déplacement et le lieu de l'action entière (doći $u N_{A c c} /$ biti $u N_{L o c}$ ). Sur le plan formel, donc, les deux langues utilisent des moyens différents pour désigner le mouvement, le français s'appuyant prioritairement sur le verbe et le serbe sur le système casuel.

\section{La conclusion}

Dans cet article nous avons analysél'impact des éléments extralinguistiques dans l'expression des relations spatiales en français et en serbe. L'analyse montre que les deux langues se ressemblent considérablement du point de vue de la 
taille de la cible et du site ainsi que concernant la position des objets par rapport à la structure axiale et les propriétés dimensionnelles du site. Le français et le serbe respectent la contrainte de localiser un objet plus petit et mobile (la cible) par rapport à un objet plus grand, stable et connu (le site). Les deux langues expriment l'orientation par rapport aux axes vertical, horizontal et frontal, le français à l'aide des prépositions directionnelles, le serbe par les cas génitif et instrumental. En français et en serbe, les propriétés dimensionnelles de surface (2dim) et le rapport d'intériorité (3dim) sont marquées par les prépositions sur$n a$ et dans- $u$. La différence la plus remarquable entre les deux langues consiste dans la conceptualisation ponctuelle exprimée par la préposition française $\grave{a}$, qui joue aussi un certain rôle dans l'expression du mouvement, alors que le serbe, par son opposition accusatif/locatif, marque sur le plan formel le trait d'aboutissement de l'action du déplacement. Les deux langues divergent donc surtout sur le plan de l'expression du mouvement.

\section{Bibliographie}

AŠIĆ 2008 : AŠIĆ, Tijana. Espace, Temps, Prépositions. Genève : Librairie Droz S.A., 2008.

BORILLO 1998 : BORILLO, Andrée. L'espace et son expression en français. Paris : Ophrys, 1998.

GOUGENHEIM 1949 : GOUGENHEIM, Georges. «L'espace à deux dimensions et l'espace à trois dimensions en français ». Journal de psychologie normale et pathologique, $\mathrm{n}^{\mathrm{o}} 17$ ?? (1949) : 35-52.

IVIĆ 1957 : IVIĆ, Milka. „Jedno poglavlje iz gramatike našeg modernog jezika - sistem mesnih padeža". Godišnjak Filozofskog fakulteta u Novom Sadu, II (1957) : 145-158. [orig.] ИВИЋ, Милка. „Једно поглавље из граматике нашег модерног језика - систем месних падежа". Годишњак Филозофског факултета у Новом Саду, II (1957) : 145158.

IVIĆ 1957-58 : IVIĆ, Milka. „Sistem predloških konstrukcija u srpskohrvatskom jeziku". Južnoslovenski filolog, vol. XXII, $\mathrm{n}^{\mathrm{o}}$ 1-4 (1957-1958) : 141-166. [orig.] ИВИЋ, Милка. „Систем предлошких конструкција у српскохрватском језику”. Јужнословенски филолог, вол. XXII, бр. 1-4 (1957-1958) : 141-166.

LANDAU, JACKENDOFF 1993: LANDAU, Barbara et Ray JACKENDOFF. "“What" and "where" in spatial language and spatial cognition". Behavioral and brain sciences, $\mathrm{n}^{\circ} 16$ (1993) : 217-265.

LAUR 1993 : LAUR, Dany. « La relation entre le verbe et la préposition dans la sémantique du déplacement ». Langages nº 110 (1993) : 47-67. 
PIPER 2001 : PIPER, Predrag. Jezik i prostor. Beograd : Čigoja štampa, 2001. PIPER, ANTONIĆ et al 2005 : PIPER, Predrag i Ivana ANTONIĆ, Vladislava RUŽIĆ, Sreto TANASIĆ, Ljudmila POPOVIĆ, Branko TOŠOVIĆ. Sintaksa savremenoga srpskog jezika. Prosta rečenica. Beograd: Institut za srpski jezik SANU - Beogradska knjiga - Matica srpska, 2005. [orig.] ПИПЕР, Предраг и Ивана АНТОНИЋ, Владислава РУЖИЋ, Срето ТАНАСИЋ, Људмила ПОПОВИЋ, Бранко ТОШОВИЋ. Синтакса савременога српског језика. Проста реченица. Београд: Институт за српски језик САНУ - Београдска књига - Матица српска, 2005.

TALMY 2000 : TALMY, Leonard. Toward a cognitive semantics. Cambridge : MIT, 2000.

VANDELOISE 1986 : VANDELOISE, Claude. L'Espace en français. Paris : Éditions du Seuil, 1986.

VANDELOISE 1988 : VANDELOISE, Claude. «Les usages spatiaux statiques de la préposition à ». Cahiers de lexicologie, $\mathrm{n}^{\circ}$ 53, (1988-2) : 119-148.

Ivana M. Vilić

\section{EKSTRALINGVISTIČKI FAKTORI U IZRAŽAVANJU SPACIJALNIH ODNOSA U FRANCUSKOM I SRPSKOM JEZIKU}

U radu su analizirani ekstralingvistički faktori u izražavanju spacijalnih odnosa u francuskom i u srpskom jeziku. Analiza pokazuje da postoje velike sličnosti u načinu izražavanja fizičkih karakteristika objekta lokalizacije i lokalizatora, kao i sličnosti u lokalizaciji u odnosu na sistem osa i dimenzionalne karakteristike lokalizatora. Oba jezika poštuju pravilo da se manji i pokretan objekat lokalizacije smešta u odnosu na veći, stabilniji i poznatiji lokalizator. U oba jezika važi princip lokalizacije u odnosu na sistem osa - vertikalnu, horizontalnu i frontalnu, pri čemu francuski jezik koristi direkcionalne predloge, dok srpski jezik lokalizaciju u odnosu na sistem osa vrši najčešće uz pomoć genitiva ili instrumentala, pri čemu genitiv izražava obeležje ograničenosti koje nije prisutno u značenju instrumentala. Dimenzionalne karakteristike lokalizatora izražene su u oba jezika na sličan način: dvodimenzionalnu površinu u oba jezika izražavaju predlozi na-sur, dok trodimenzionalnu zapreminu izražavaju predlozi $u$-dans. Najveća razlika između francuskog i srpskog jezika u pogledu ekstralingvističkih faktora lokalizacije u prostoru uočava se u načinu punktuelne konceptualizacije mesta, što je u francuskom izraženo predlogom à, dok srpski svojom opozicijom akuzativ / lokativ izražava ishodišnu tačku radnje kretanja (akuzativ) u odnosu na mesto čitavog toka radnje (lokativ). Budući da opozicija akuzativ / lokativ u srpskom jeziku najčešće učestvuje u izražavanju dinamičkog aspekta lokalizacije u prostoru, odnosno kretanja, a da se kretanje i 